\title{
Liquen plano: Revisión de la literatura actual
}

\section{Oral lichen planus. A review of literature}

\author{
Matesanz-Pérez P*, Bascones-Martínez, A**
}

\section{RESUMEN}

Objetivo: Proporcionar información actualizada acerca del liquen plano oral, prestando atención especial a su posible malignización y a su relación con el cáncer oral.

El liquen es una enfermedad mucocutánea con una relevancia particular en la cavidad oral, no sólo debida a su prevalencia, sino también debida al hecho de estar englobada dentro de los denominados "enfermedades o estados premalignos".

La relación del liquen plano con el cáncer oral aumenta la necesidad de revisar la literatura, para así comprender cuánto puede hacer el clínico al respecto, y aprender cómo manejar esta patología.

Palabras clave: Liquen plano oral; apoptosis; cáncer oral; tratamiento; inmunología; linfocitos.

\section{SUMMARY}

Aims: To provide updated knowledge about oral lichen planus, paying especial attention to its possible malignant transformation and the relationship with oral cancer.

Oral lichen planus is a mucocutaneous disorder with particular relevance in the oral cavity, not only due to its prevalence, but due to the fact of belonging to the group of "premalignant lesions or states".

The relationship that this pathology has with oral cancer increases the need for literature reviewing so as to learn what the practitioner can do about it and how to manage it.

Key words: Oral lichen planus; apoptosis; oral cancer; treatment; immunology; lymphocytes.

Fecha de recepción: 15 de septiembre de 2008.

Aceptado para publicación: 17 de septiembre de 2008.

* Máster Periodoncia UCM.

** Catedrático de Medicina Oral y Periodoncia UCM.

Matesanz-Pérez P, Bascones-Martínez, A. Liquen plano: Revisión de la literatura actual. Av. Odontoestomatol 2009; 25 (2): 99-114.

\section{INTRODUCCIÓN}

El liquen plano oral es un desorden del epitelio escamoso que cobra importancia por dos motivos principalmente: por un lado está su frecuente aparición en todo el mundo, especialmente en sujetos de edad media, con mayor incidencia en el sexo femenino (1). Por otro lado, el liquen plano debe ser estudiado con cuidado dado su potencial de malignización. A pesar de la permanente controversia entre las distintas fuentes a la hora de establecer las probabilidades de que el liquen oral sufra una transformación ma- 
ligna, la OMS, en su último volumen acerca de los tumores de cabeza y cuello, recomienda el desarrollo de criterios diagnósticos que permitan diferenciar el liquen plano oral (LPO) de las lesiones liquenoides orales (OLL), pero se menciona que ambas entidades deben considerarse susceptibles de sufrir una degeneración maligna hasta que dichos criterios estén disponibles (2).

El objetivo de este trabajo es doble. En primer lugar se pretende revisar la literatura más reciente acerca de este tipo de lesiones con la idea de actualizar el conocimiento que de ellas se tiene, pero con el objetivo adicional de profundizar en la evidencia científica que explica cómo desde una lesión benigna, de curso asintomático en la mayoría de los casos, se puede pasar a una lesión de carácter maligno, con un pronóstico más incierto.

\section{EPIDEMIOLOGÍA}

Tal y como se recoge en todos los tratados de Medicina Oral, el LPO se trata de la enfermedad mucocutánea más frecuente de la cavidad oral, sin embargo resulta difícil dar un dato de prevalencia exacta, ya que los números varían en función de la región geográfica que se estudie.

En general se habla de prevalencias alrededor del $0.5-2 \%$ (3) en todo el Mundo, y poco varían estos números si se centra la atención en el área del Mediterráneo. En un análisis reciente de la prevalencia de lesiones de la mucosa oral llevado a cabo en Turín (Italia), se estudió la frecuencia de gran cantidad de entidades clínicas, entre las que se encontraban el liquen plano oral y las reacciones liquenoides. Ambas patologías fueron estudiadas en función de la edad, el sexo, y ciertos factores de riesgo asociados (Tablas 1 a 4).

De estos datos, así como a partir de otras publicaciones europeas de los últimos años, se extrae que el liquen plano oral aparece más frecuentemente en mujeres, generalmente en la edad adulta, y su prevalencia es mayor en sujetos de raza blanca $(5,6)$.

En un estudio de 2006, se vio que en una población de 690 individuos en estados Unidos, la edad media de aparición del LPO fue 52 años (53 años en muje-

\section{TABLA 1.- LPO EN RELACIÓN CON LOS FACTORES DE RIESGO TABACO Y ALCOHOL (4)}

\begin{tabular}{|l|c|c|c|c|c|}
\hline Sexo & $\begin{array}{c}\text { Fumadores } \\
\text { leves }\end{array}$ & $\begin{array}{c}\text { Muy fumadores } \\
\text { (>10 cigs) }\end{array}$ & $\begin{array}{c}\text { Fumadores } \\
\text { y exfumadores }\end{array}$ & Alcohol & Tabaco+alcohol \\
\hline$\sigma$ & 0,32 & 3,06 & 1,23 & 1,14 & 0,00 \\
\hline o & 1,17 & 2,02 & 1,03 & 1,76 & 1,60 \\
\hline TOTAL & 0,70 & 1,72 & 1,15 & 1,30 & 0,40 \\
\hline
\end{tabular}

\section{TABLA 2.- REACCIONES LIQUENOIDES EN RELACIÓN CON LOS FACTORES DE RIESGO TABACO Y ALCOHOL (4)}

\begin{tabular}{|l|c|c|c|c|c|}
\hline Sexo & $\begin{array}{c}\text { Fumadores } \\
\text { leves }\end{array}$ & $\begin{array}{c}\text { Muy fumadores } \\
\text { (>10 cigs) }\end{array}$ & $\begin{array}{c}\text { Fumadores } \\
\text { y exfumadores }\end{array}$ & Alcohol & Tabaco+alcohol \\
\hline$\sigma$ & 0,47 & 0,83 & 0,33 & 0,18 & 0,80 \\
\hline o & 0,19 & 0,00 & 0,39 & 0,50 & 0,00 \\
\hline TOTAL & 0,35 & 0,34 & 0,35 & 0,39 & 0,60 \\
\hline
\end{tabular}




\begin{tabular}{|l|c|c|c|}
\hline \multicolumn{4}{|c|}{ TABLA 3.- LPO EN RELACIÓN CON } \\
LA EDAD (4) \\
\hline Sexo & $<40$ años & $40-60$ años & $>60$ años \\
\hline$\sigma$ & 3,29 & 1,13 & 1,39 \\
\hline o & 0,22 & 1,03 & 2,43 \\
\hline TOTAL & 0,40 & 1,13 & 2,94 \\
\hline
\end{tabular}

\section{TABLA 4.- REACCIONES LIQUENOIDES EN RELACIÓN CON LA EDAD (4)}

\begin{tabular}{|l|c|c|c|}
\hline Sexo & $<40$ años & $40-60$ años & $>60$ años \\
\hline$\sigma^{\nearrow}$ & 0,22 & 0,41 & 0,16 \\
\hline O & 0,22 & 0,41 & 0,37 \\
\hline TOTAL & 0,10 & 0,41 & 0,26 \\
\hline
\end{tabular}

res y 48 en hombres), con un rango entre los 16-53 años. La mayoría de los pacientes desarrollaron las lesiones en la quinta o sexta década de la vida, y menos de un $1 \%$ lo hicieron antes de los 20 años. Parece que vieron una cierta tendencia a la aparición de las lesiones en hombres a edades más tempranas que en las mujeres (7).

\section{ETIOLOGÍA}

Al hablar de la etiología del liquen plano oral debe hacerse una diferenciación entre aquellos factores

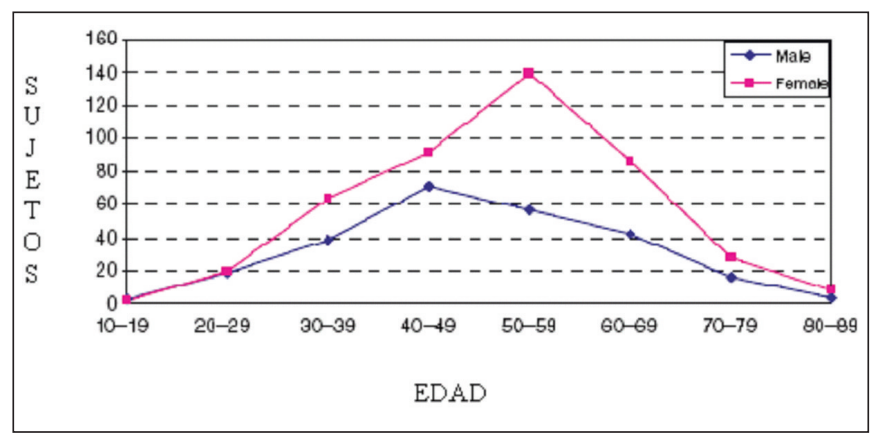

Gráfico 1. que predisponen a su padecimiento, y aquellos factores que lo acaban desencadenando. Para un análisis más sencillo de los agentes etiológicos, pueden clasificarse como factores predisponentes y factores desencadenantes.

\section{FACTORES PREDISPONENTES}

La existencia en una misma familia de sujetos con liquen plano oral induce a sospechar de una posible predisposición de carácter hereditario. Esta mayor susceptibilidad podría estar condicionada por los antígenos de histocompatibilidad, (HLA-A3, HLA-B7, HLA-A28, HLA-DR1, DQW1), o por su expresión en las células de Langerhans.

\section{FACTORES DESENCADENANTES}

Existen diferentes motivos por los que puede acabar desarrollándose un liquen plano. De hecho, muchos de ellos son inciertos, y en ocasiones se habla de esta enfermedad como de causa idiopática, ya que es difícil precisar a qué es debida. En cualquiera de los casos, la literatura sí recoge diversas explicaciones que justificarían su aparición.

\section{a. Factores psíquicos}

En ocasiones se habla del LPO como de una alteración dermatológica de causa psicosomática o de un problema neuroinmunológico con afectación cutánea.

Se sabe que existen sujetos hipersensibles, irritables y nerviosos, con LPO, en los que es necesario encontrar el equilibrio psicosomático para la resolución del problema, y en los que el estrés actúa como factor precipitante del cuadro. En estos mismos sujetos, la ansiedad o el factor emocional sería capaz de cronificar la patología, o condicionar la aparición de las formas clínicas rojas, más dolorosas y complicadas que las blancas (8). Por lo tanto, además de ser un posible factor de riesgo, a ansiedad también agravaría a lesión (9).

Se ha relacionado el estado de ánimo con la situación inmunológica y los niveles de cortisol, especu- 
lándose sobre la posibilidad de que una alteración en el estado de ánimo pueda producir un impacto sobre el sistema neuroendocrino, que influiría en las subpoblaciones linfocitarias de los pacientes con LPO (8).

\section{b. Fármacos}

La ingesta de determinados fármacos puede desencadenar la aparición de LPO o de cuadros que, por su similitud clínica con éste, se denominan reacciones o lesiones liquenoides, pero que poseen un curso diferente al liquen (10). Las diferencias entre ambos tipos de patología resultan difíciles de identificar con la exploración clínica, y en ocasiones la histología de las lesiones tampoco proporciona una gran ayuda para el diagnóstico.

El periodo de latencia desde el contacto o la ingesta del fármaco hasta la aparición de la lesión son variables, desde 10 días hasta varios años, y tan sólo la desaparición de las lesiones tras retirar el tratamiento farmacológico confirmaría el diagnóstico de reacción liquenoide.

Son muchos los fármacos que pueden dar lugar a este tipo de cuadros: el oro, los antipalúdicos, la penicilina, los diuréticos, los $\beta$-bloqueantes, las quininas, los AINEs, los IECAs, etc, son algunos de los posibles ejemplos (10).

\section{c. Materiales odontológicos}

Del mismo modo que los fármacos, el contacto con determinados materiales de restauración puede originar lesiones indistinguibles clínicamente del LPO, pero que sin embargo deben entenderse como reacciones liquenoides. Dentro de estos materiales cabe destacar la especial participación de la amalgama de plata, que con frecuencia se asocia a la aparición de lesiones blanquecinas reticulares con aspecto arboriforme (11-14).

Se puede entender la lesión liquenoide reactiva al contacto con la amalgama como una alergia de contacto a este material. Por este motivo aparece generalmente en contacto directo con una obturación, lo que permite confirmar el diagnóstico siem- pre que el paciente no presente al mismo tiempo otras lesiones propias del LPO. La sustitución de este material por otro, como las resinas compuestas, ayuda a la resolución del cuadro (14).

\section{d. Factores microbiológicos}

Ya se ha mencionado la posibilidad de que las lesiones de tipo atrófico-erosivo puedan sobreinfectarse y complicarse, sin embargo, algunos autores hablan de determinados microorganismos como posibles agentes causales de las lesiones originales (15-17).

En un consenso sobre el liquen plano oral en 2005, se habló de varios virus posiblemente implicados en la etiopatogenia de la enfermedad (16). Entre ellos se menciona el virus del herpes humano, el virus de la inmunodeficiencia humana, el papilomavirus y el mencionado con mayor frecuencia en la literatura, el virus de la hepatitis $\mathrm{C}$.

Dentro de la familia del virus del herpes humano se ha establecido una posible asociación con el tipo 1 , el 6, el citomegalovirus y el virus de Epstein-Barr. Todos ellos suelen asociarse a las formas rojas de LPO, y aún no está claro si la asociación es causal, o si se trata más bien de una sobreinfección vírica de las lesiones (16).

En cuanto al VIH, no se sabe si es el propio virus el que induce la enfermedad, o si más bien es el tratamiento farmacológico que reciben estos pacientes el causante de lesiones similares a las del LPO, pero que en realidad se trata de lesiones de tipo liquenoide.

Del virus del papiloma se han emitido pocas afirmaciones definitivas, ya que hay gran cantidad de variabilidad en los datos publicados acerca de la asociación con el liquen. Sin embargo, el virus de la hepatitis C parece ser el más estudiado y el más claramente asociado a esta enfermedad mucocutánea (16).

\section{e. Factores inmunológicos}

Cada día se desarrolla más la evidencia científica que defiende el papel de la alteración inmunológica 
como eje de la patogénesis del LPO, concretamente en lo que a la inmunidad celular se trata. La implicación de los linfocitos $\mathrm{T}$ y de las células presentadoras de antígeno es uno de los aspectos más defendidos en la literatura actual (10). Se ha planteado la posibilidad de que la actividad inmune de estos pacientes esté alterada, aunque también se han planteado otras opciones, como la posible presencia de clones de células $T$ autocitotóxicas, tal y como se ha podido observar en la histología de las lesiones de esta enfermedad (18).

Algunos autores hablan del LPO como de una enfermedad inflamatoria crónica caracterizada por la respuesta de las células $T$ en contra de las células epiteliales, lo que conlleva una acumulación de linfocitos T y de células epiteliales dañadas (19). Como consecuencia de este desajuste de las células $\mathrm{T}$, se va a alterar el balance de citoquinas producidas por éstas. Los cambios humorales que tienen lugar en los pacientes con LPO, por consiguiente, tendrían cierto valor potencial en la monitorización de los pacientes con liquen previa y posteriormente al tratamiento (20).

El aspecto que mayores dudas plantea en los últimos tiempos es la naturaleza del antígeno que despierta la actividad de estos clones celulares. De acuerdo con el metaanálisis de 2005, cabe la posibilidad de que se trate de un péptido endógeno, en cuyo caso el LPO debería entenderse como una enfermedad autoinmune y lo cierto es que existen ciertas características en la forma de presentación de la enfermedad que dan sentido a esta hipótesis: la cronicidad, la aparición a edades adultas y la asociación con otras enfermedades de carácter autoinmune (16).

Por otro lado, se han planteado otras alternativas, como la posibilidad de que fueran las heat shock proteins, que se encuentran aumentadas en los queratinocitos del LPO las que causaran la hiperrespuesta de los linfocitos (16).

\section{f. Otros}

El tabaco y la diabetes mellitus, por ejemplo, aunque por el momento no se ha demostrado su participación en el desarrollo de las lesiones iniciales del LPO, sí podrían actuar sobre el epitelio ya afectado previamente, agravando la situación original (21).

\section{CLÍNICA}

Se ha hablado del LPO como de una lesión en forma de "puntos, líneas y rayas de color blanco que dibujan en conjunto una red o retículo dendriforme similares a las hojas del helecho, a un encaje o a mosaicos".

Aunque el LPO aparezca con mayor frecuencia en la mediana edad, es un padecimiento que también puede desarrollarse en niños y jóvenes, en cuyo caso van a aparecer lesiones de iguales características y evolución que en sujetos de mayor edad (22). En todos los casos, aunque las lesiones que el odontólogo puede identificar primariamente se localizan en la cavidad oral, es posible que se acompañen de otras localizaciones, como las uñas, la piel u otras mucosas. Las lesiones cutáneas pueden darse hasta en un $30-50 \%$ de los casos, por lo que su identificación puede ayudar al diagnóstico de esta entidad (3).

\section{Lesiones cutáneas}

La lesión típica de la piel consiste en la aparición de un área poligonal, no elevada, de coloración violácea, que suele acompañarse de un cierto prurito, y que con frecuencia van a encontrarse en áreas de flexión. A menudo, estas placas cutáneas acompañan a las "uñas en papel de fumar", y a la descamación del cuero cabelludo (23).

Las uñas se hiperqueratinizan y se engrosan. Sin embargo, se vuelven frágiles y presentan grietas, por lo que pueden romperse con facilidad.

En el caso del cuero cabelludo, puede aparecer una destrucción progresiva del folículo que, en caso de no tratarse, puede dejar áreas de cicatrización con alopecia.

\section{Afectación de la mucosa oral}

Las lesiones que aparecen en la mucosa oral se van a caracterizar por el pleomorfismo; es decir, la posi- 


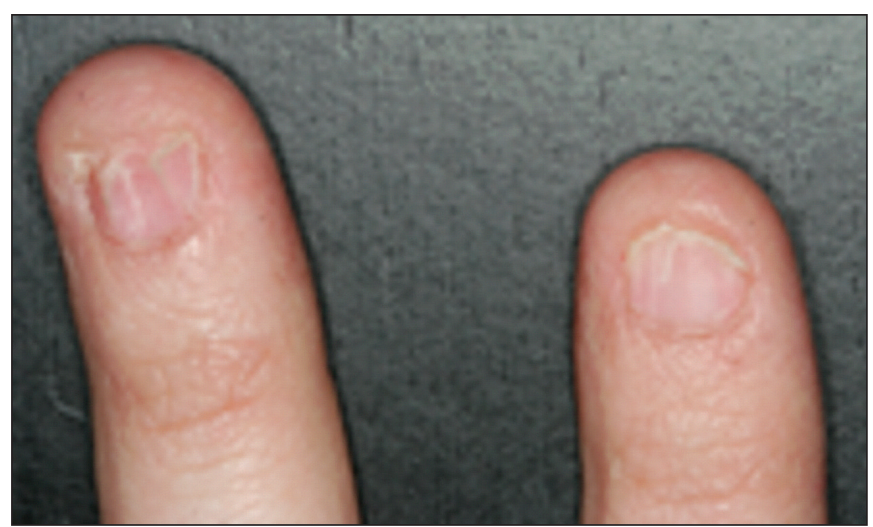

Fig. 1.

bilidad de adoptar diferentes conformaciones y características, que pueden complicar su identificación. A pesar de su variabilidad, habitualmente se clasifican dentro de dos grandes grupos: formas clínicas blancas y formas clínicas rojas. Dentro de estas formas, también es posible identificar distintas entidades clínicas, como a continuación se revisa.

\section{Formas blancas: Liquen plano reticular y en placas}

A este apartado pertenece la lesión más típica del LPO, con forma de estrías blanquecinas que no se desprenden con el raspado, y que se entrecruzan

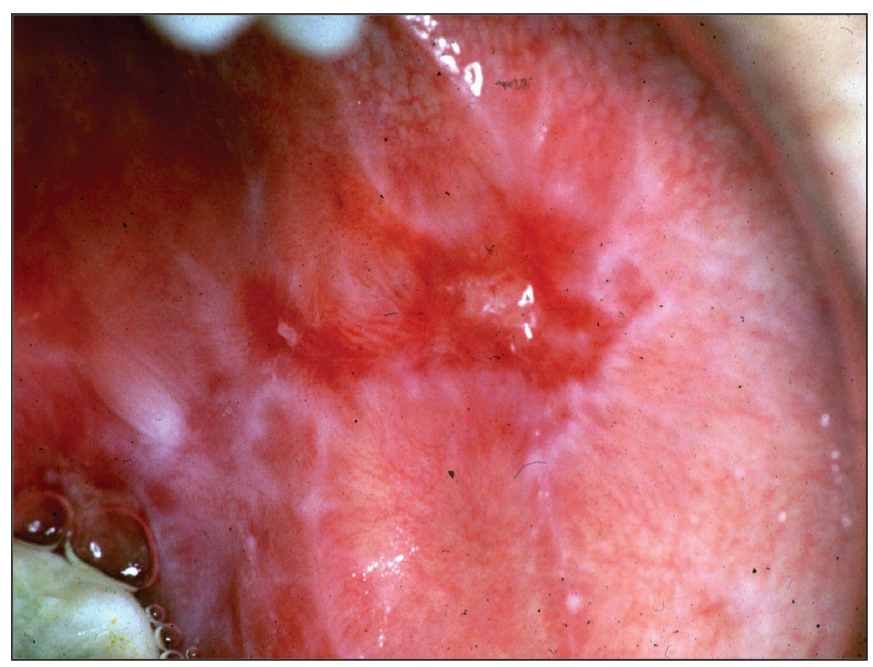

Fig. 2. originando formas arboriformes o estrelladas con un fondo que puede ser normal o eritematoso (23). Esta lesión estriada recibe el nombre de estrías de Wickham, y suelen ser asintomáticas, por lo que su hallazgo suele ser casual.

Se localizan en la mayoría de los casos en la mucosa yugal, y generalmente suelen ser bilaterales (22). Sin embargo, también es posible encontrar imágenes de este tipo en otras zonas de la mucosa oral, tales como la lengua, la encía, la mucosa labial o el paladar.

Por otro lado, al igual que la forma anterior, el LPO en placas suele ser asintomático. Se asemeja clínicamente a la leucoplasia, ya que se trata de una placa blanquecina que tampoco puede desprenderse mediante raspado. Sin embargo, a diferencia de ésta, las lesiones en placas del LPO suelen ser multifocales (23).

Lesiones de este tipo van a localizarse en el dorso de la lengua y en la mucosa bucal.

\section{Formas rojas: Liquen plano erosivo y atrófico}

A diferencia de las formas blancas, las variantes rojas del LPO suelen cursar con diferentes grados de

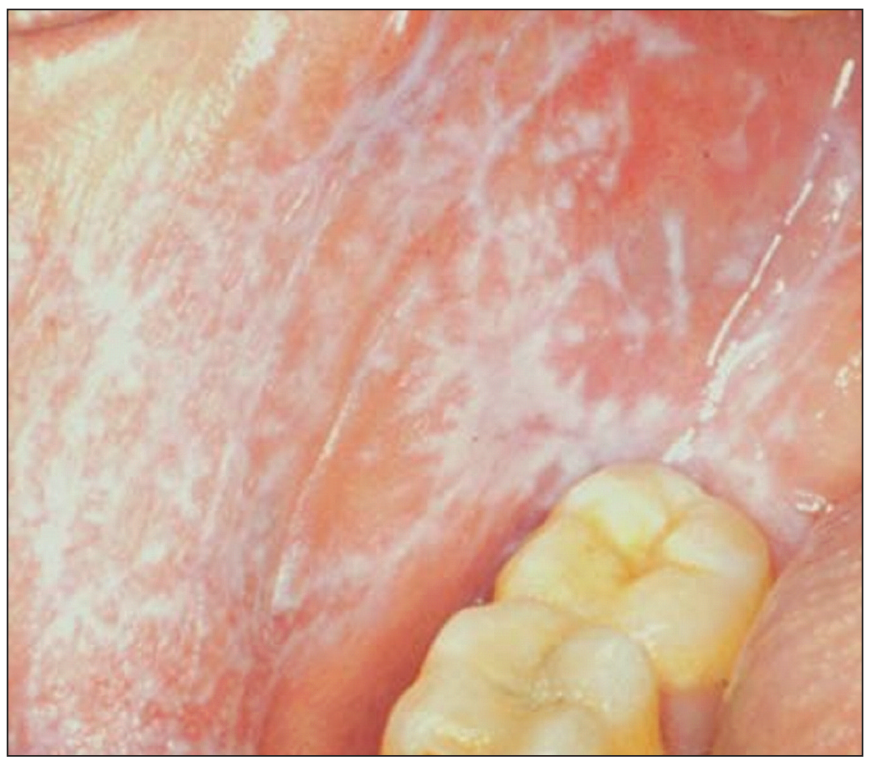

Fig. 3. 
dolor y disconfort. En estas formas, el paciente presenta un número variable de úlceras y ampollas por la mucosa oral, que rápidamente se rompen y dejan áreas de erosión dolorosas que pueden llegar a interferir con las funciones de masticación y deglución $(22,23)$.

La forma erosiva es la segunda forma más frecuente. Cursa con una mezcla de áreas de ulceración rodeadas por finas estrías, y puede evolucionar hacia la forma atrófica. En ella puede identificarse una zona rosácea, eritematosa, consecuencia del adelgazamiento del epitelio y a la posterior transparencia de los vasos sanguíneos a través de éste.

Las formas rojas no suelen curar espontáneamente, y pueden confundirse con otras alteraciones de la mucosa, como ciertos cuadros de origen autoinmune, con los que comparten las características clínicas (22).

Las formas rojas pueden acompañarse de cierto grado de xerostomía, lo que hace posible la sobreinfección de las lesiones y complica aún más su resolución.

Además de estas formas clásicas, otros autores han descrito otras formas de presentación de esta enfermedad mucocutánea. Entre ellas puede hablarse de: liquen zooniforme, anular, ampollar, vegetante, nigricans, etc.

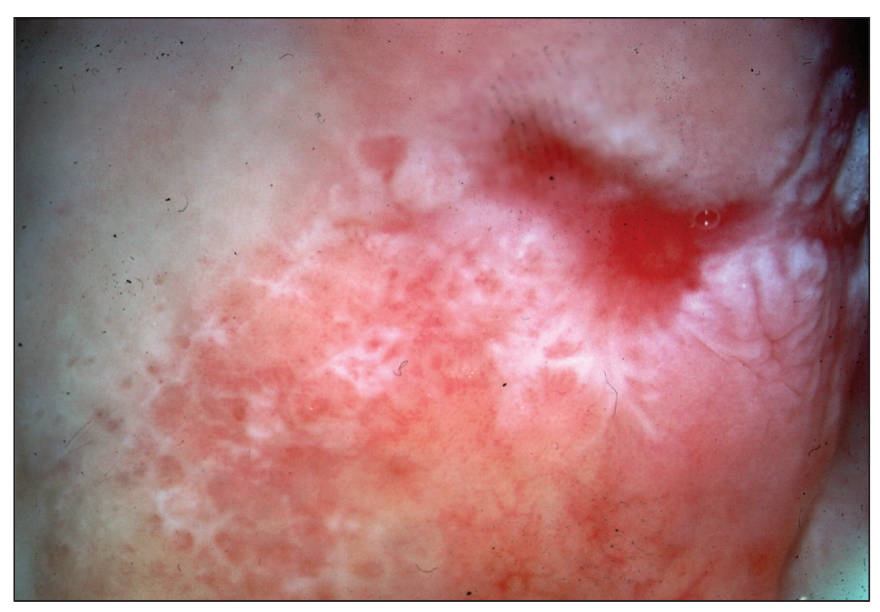

Fig. 4.

\begin{tabular}{|l|c|c|c|}
\hline \multicolumn{4}{|c|}{ TABLA 5 } \\
\hline $\begin{array}{l}\text { Formas } \\
\text { clínicas }\end{array}$ & $\begin{array}{c}\text { Total } \\
\mathbf{( \% )}\end{array}$ & $\begin{array}{c}\text { Hombres } \\
\mathbf{( \% )}\end{array}$ & $\begin{array}{c}\text { Mujeres } \\
\text { (\%) }\end{array}$ \\
\hline Lesiones blancas & 42,7 & 17,1 & 28,9 \\
\hline Reticular & 30,5 & 20 & 80 \\
\hline En placas & 12,2 & 10 & 90 \\
\hline Lesiones rojas & 25,6 & 23,8 & 76,2 \\
\hline Atrófico & 2,4 & 50 & 50 \\
\hline Erosivo & 23,2 & 21,1 & 78,9 \\
\hline Mixto & 31,7 & 15,4 & 84,6 \\
\hline Total & 100 & 100 & 100 \\
\hline
\end{tabular}

En general, un aspecto que caracteriza a todas estas lesiones es la posibilidad de que se transformen, de manera que unas formas clínicas den lugar a otras. La permanente vigilancia de las lesiones es fundamental, para de este modo poder identificar cualquier posible transformación maligna del LPO.

En algunas ocasiones, ambas formas clínicas, rojas y blancas, pueden aparecer en un mismo sujeto. La mayor o menor incidencia de las distintas formas clínicas aparece recogida en publicaciones de los últimos años y parece estar en función del sexo (24).

\section{Afectación de otras mucosas}

El esófago, la laringe, el esófago, la conjuntiva o el ano, así como la vulva o el pene, son otras áreas de común asentamiento del liquen plano. De hecho se habla de una afectación plurimucosa o síndrome vulvovaginal, que aparece en un $20 \%$ de mujeres afectadas por el liquen (25). En ellas, además de las clásicas lesiones orales, pueden encontrarse signos y síntomas en otras mucosas, acompañados de un intenso prurito, quemazón y cierto dolor (1).

\section{Gingivitis descamativa}

Este término clínico se emplea para la manifestación gingival de las enfermedades mucocutáneas, y 
está caracterizado por la aparición de una encía eritematosa, brillante, friable y con tendencia al sangrado, que puede acompañarse de dolor (26). No se trata de una entidad patológica en sí, sino que suele considerarse más bien un signo clínico de otro cuadro (27).

Esta forma clínica puede aparecer en pacientes con LPO, sin embargo no es patognomónico de esta entidad. Las tres enfermedades en las que aparece la gingivitis descamativa con mayor frecuencia son: el penfigoide cicatricial, el liquen plano erosivo y el pénfigo. Sin embargo, a pesar de la predilección de la gingivitis descamativa por estos cuadros, es posible detectarla en combinación con otras patologías, como: penfigoide bulloso, estomatitis de contacto, epidermólisis ampollar. La dificultad que entraña el diagnóstico acertado de estas patologías complica la identificación de la gingivitis descamativa, lo que en ocasiones interfiere en la elección del tratamiento adecuado y en la evolución (28).

\section{DIAGNÓSTICO E HISTOPATOLOGÍA}

Para el correcto diagnóstico del liquen plano oral es necesaria la correlación entre los hallazgos clínicos y los histopatológicos; esto es, la identificación de lesiones de patrón reticular o de aspecto atrófico-erosivo debe estar acompañada de un análisis histológico que descarte la displasia epitelial (29). En casi un $25 \%$ de casos, se va a producir una discrepancia entre el diagnóstico clinicopatológico y el histopatológico (30).

La Organización Mundial de la Salud elaboró recientemente una propuesta de criterios diagnósticos que deben cumplirse para garantizar la identificación del LPO y de las reacciones liquenoides (31)

A pesar de que con estos criterios se pensaba hacer más unánime el diagnóstico de estas lesiones, se ha visto que todavía existe cierta discrepancia inter e intraobservador a la hora de hacer el diagnóstico $(32,33)$. Tanto los aspectos clínicos como los detalles histológicos, a pesar de haber sido definidos con bastante precisión, están sujetos a cierta valoración subjetiva, por lo que en ocasiones es difícil diferen- ciar las lesiones de las provocadas por otras patologías.

En 1978 la OMS formuló una definición histopatológica para facilitar el diagnóstico (31): "Los rasgos histopatológicos del LPO son característicos. Suele haber una capa de queratosis, bien orto o paraqueratosis. Si la queratinización es normal en la zona afectada, entonces la capa de queratina aparecerá

\section{PROPUESTA DE DIAGNÓSTICO DE LA OMS}

\section{Criterios clínicos}

- Presencia de lesiones bilaterales más o menos simétricas.

- Presencia de entramado reticular de color blanco-grisáceo (patrón reticular).

- Lesiones erosivas, bullosas o en placas sólo se aceptan como subtipo en presencia de lesiones reticulares en otra localización de la mucosa

- En todo el resto de situaciones que se asemejen, pero no coincidan exactamente con estos criterios, debe emplearse el término de "clínicamente compatible con LPO”.

\section{Criterios histopatológicos}

- Presencia de una banda bien definida de infiltrado celular confinada a la zona más superficial del tejido conectivo, basada principalmente en linfocitos.

- Signos de degeneración hidrópica de la capa basal.

- Ausencia de displasia epitelial.

- En caso de signos histopatológicos me nos obvios, debe emplearse el término de "histológicamente compatible con LPO”.

Diagnóstico final de LPO o lesiones liquenoides orales (LLO)

- LPO $\rightarrow$ Cumplimiento de ambos apartados

- LLO $\rightarrow$ Se emplea este término en los siguientes casos:

1. Clínica similar a la del LPO, pero histopatológicamente "compatible con LPO".

2. Histopatológicamente coincidente con el LPO pero clínicamente "compatible con LPO".

3. Clínica e histopatológicamente "compatible con LPO". 


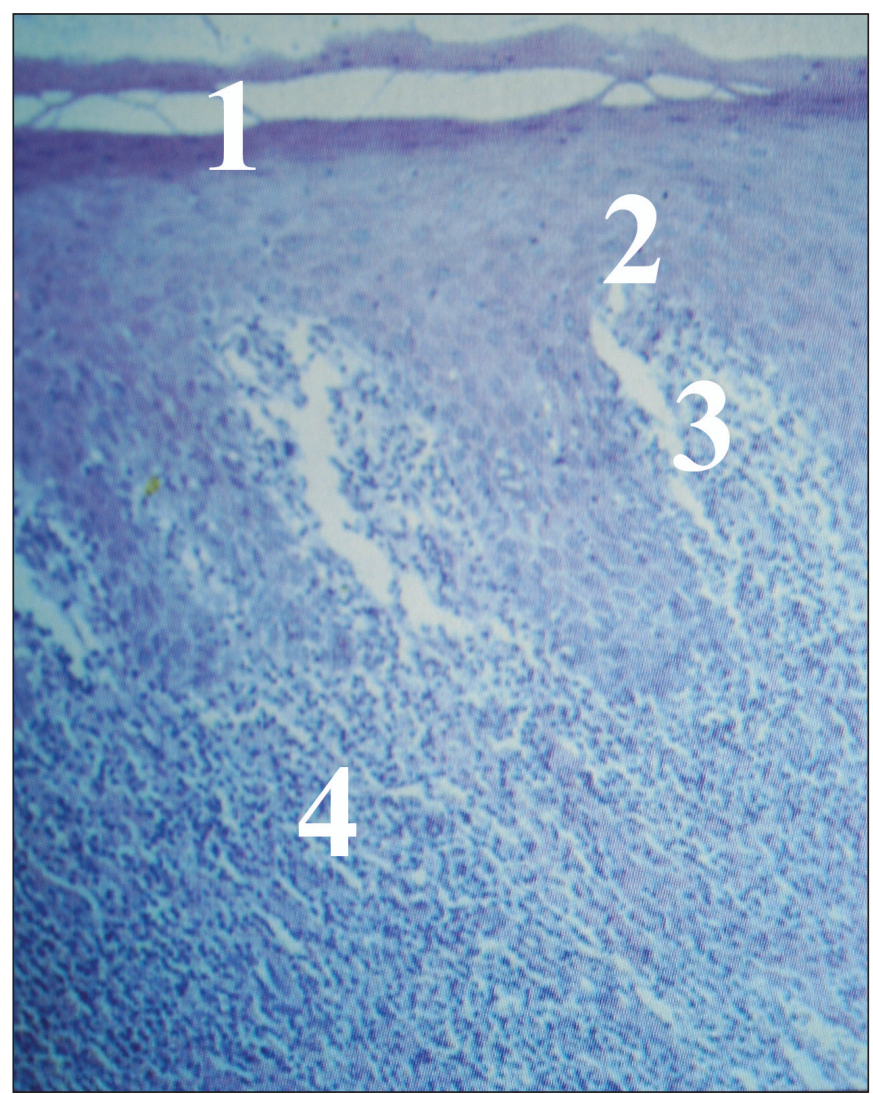

Fig. 5. 1: Hiperqueratosis; 2: Acantosis y granulosis; 3: Degeneración hidrópica de la capa basal; 4: Infiltrad inflamatorio en banda.

engrosada. Sin embargo, si la zona no debería presentar queratinización en condiciones normales, como por ejemplo en la mucosa bucal, entonces la capa de queratina será fina. Del mismo modo ocurre con el estrato granulosos; en caso de ser típico de la zona afectada, en los casos con LPO habrá un engrosamiento, mientras que de no ser así, la capa de células granulares será fina. El grosor del epitelio puede variar, y en ocasiones se aprecia atrofia de éste.

Pueden estar presentes los característicos cuerpos de Civatte (coloidales) en la zona de la capa basal, bien en el epitelio, o en la parte más superficial del tejido conectivo. Se trata de estructuras acidófilas de forma redondeada o lobulada, que en ocasiones contienen núcleos picnóticos o fragmentos nucleares.

Los cambios de las células de la capa basal incluyen "degeneración hidrópica", y puede verse una banda estrecha de material eosinófilo sobre la membrana basal.

Se va a ver una zona bien definida de infiltrado inflamatorio confinada a la parte superficial del tejido conectivo (lámina propia), que consiste principalmente de linfocitos" (31).

Aunque se trate de una definición precisa, con mucho detalle, en realidad, en el proceso que sigue el patólogo para hacer el diagnóstico es relativamente subjetivo. En él influyen su experiencia, entrenamiento, y la información que le haya proporcionado el clínico (32).

La degeneración hidrópica de la capa basal se ha entendido clásicamente como un signo histomorfológico del proceso de apoptosis celular que tiene lugar en el liquen (34). Dicha degeneración incluye una condensación de la cromatina en la periferia de núcleo, acompañada de picnosis nuclear y desintegración nucleolar. Sin embargo, existen autores que consideran que la apoptosis en el liquen tiene poca importancia desde un punto de vista cuantitativo, por lo que la idea de que la degeneración hidrópica es responsabilidad de los mecanismos apoptóticos dejaría de tener sentido (35-37).

Los mecanismos moleculares que refutan esta asociación se han revisado en la literatura más reciente, tal y como se presenta más adelante.

Para que el análisis histopatológico pueda servir de ayuda al diagnóstico, una biopsia debe tomarse previamente, prestando atención de hacerlo siempre en un área que incluya tejido sano y preferiblemente en zonas donde las lesiones presenten aspecto reticular.

\section{Inmunofluorescencia}

Se trata de una herramienta que también puede ayudar a dilucidar el diagnóstico. Con esta prueba, en casos de LPO va a observarse el depósito de fibrinógeno a lo largo de la membrana basal, el depósito de inmunoglobulina $M$, así como un fenómeno exudativo inespecífico. La prueba de la inmunofluorescencia tiene una sensibilidad del $70 \%$ y una especificidad del $78 \%$. 


\section{MALIGNIZACIÓN DEL LPO}

La evolución del LPO es crónica con periodos de exacerbación y remisión. En cuanto a su posible potencial de malignización existe mucha controversia, aunque existe bastante consenso acerca de cuáles son las formas con mayores posibilidades de evolución hacia una lesión maligna (38).

En la literatura ya se han revisado los autores que, como Hallopeu, desde principios del siglo pasado empezaban a hablar de una posible evolución maligna de esta entidad. Sin embargo otros autores aseguran que las publicaciones que hablan de elevadas tasas de malignización se deben a un diagnóstico incorrecto del LPO, o a una población sesgada, expuesta a muchos factores de riesgo (39). Con posterioridad a estas publicaciones, se han revisado los criterios diagnósticos de esta enfermedad mucocutánea, y finalmente se ha acordado que, aunque es necesario el desarrollo de criterios diagnósticos que permitan diferenciar el LPO de las lesiones liquenoides orales (OLL), ambas lesiones deben considerarse como potencialmente malignizables hasta que la diferenciación esté clara.

Existe una base científica que podría explicar la predisposición del epitelio hacia la transformación maligna (2). Sin embargo, algunos autores ven necesario el establecimiento de un consenso en torno a ciertos temas, como son: la tasa real de malignización del LPO, los factores de riesgo para la cancerización, la influencia del tratamiento con inmunosupresores en el LPO, y el manejo clínico ideal para estos pacientes (2).

Tal y como se explicaba en el apartado anterior, las características fundamentales e nivel histopatológico del LPO son, por un lado, el infiltrado en banda de tipo intenso subyacente a la capa basal del epitelio y, por otra parte, la degeneración vacuolizante de la capa basal (40). Precisamente el infiltrado inflamatorio crónico podría entenderse como el primer factor de riesgo para la malignización, tal y como ocurre en otras áreas del cuerpo, como en la colitis ulcerosa o en la gastritis atrófica, entre otras (35, 41). De acuerdo con esta explicación, algunas moléculas y radicales producidos por las células inflamatorias pueden actuar como agentes mutagénicos para las células epiteliales o ejercer una importante influencia sobre los mecanismos de regulación del ciclo celular, como la apoptosis $(38,41)$.

\section{Mecanismos mutagénicos de las células epiteliales en el LPO}

Las células inflamatorias del LPO producen un exceso de óxido nítrico, el cual reacciona con el $\mathrm{O}_{2}$ e

\begin{tabular}{|l|c|c|c|c|c|}
\hline \multicolumn{7}{|c|}{ TABLA 6} \\
\hline Autor & Año & País & $\mathbf{n}^{\text {o }}$ LPO & \% T.M & Seguimiento \\
\hline Voute & 1993 & G. Bretaña & 241 & 3,3 & ¿ं? \\
\hline Barnard & 1993 & Israel & 280 & 2,1 & ¿? \\
\hline Mancarz & 1996 & Israel & 157 & 1,3 & 1,5 \\
\hline Gorsky & 1997 & Grecia & 326 & 1,3 & 4,8 \\
\hline Markopoulos & 1997 & E.E.U.U. & 95 & 3,2 & 6,1 \\
\hline Silverman & 1998 & Italia & 263 & 4,9 & 5,7 \\
\hline Lo Muzio & 1999 & G.Bretaña & 832 & 0,8 & 11,0 \\
\hline Rajentheran & 2001 & Italia & 502 & 3,6 & ¿ं? \\
\hline Chainani-Wu & 2001 & E.E.U.U. & 229 & 1,7 & ¿? \\
\hline Eisen & 2002 & E.E.U.U. & 723 & 0,8 & 4,5 \\
\hline
\end{tabular}


induce la formación de compuestos carcinogénicos y mutagénicos para el DNA. Por otro lado, la producción de ciclooxigenasa-2 (COX-2) por parte de estas mismas células interviene en el metabolismo del ácido araquidónico y conlleva la producción de metabolitos que también pueden dañar el DNA (41).

\section{Apoptosis en el LPO}

La apoptosis es un fenómeno de gran trascendencia por el que las células mueren tomando parte activa en su propia eliminación. Se trata de un proceso fisiológico que se activa por medio de la acción de proteasas específicas y de endonucleasas, entre los que destaca la actividad de las caspasas, particularmente la Caspasa-3, que activa y rompe otras caspasas, responsables todas ellas al final de la proteólisis.

El ataque a las células epiteliales por parte de los linfocitos T que tiene lugar en el LPO despierta la necesidad de emplear una serie de mecanismos moleculares diseñados a detener el ciclo celular, a inducir la senescencia celular, o a inducir la apoptosis para, de este modo, poder eliminar aquellas células con el DNA alterado $(34,42)$. Sin embargo, a pesar del intenso daño que son capaces de producir los linfocitos en estos casos, se observan pocos fenómenos de apoptosis que se observan en el LPO (2). Trabajos de revisión, así como trabajos experimentales describen, tras el análisis de los marcadores de la apoptosis en los queratinocitos epiteliales; es decir, tras la cuantificación de Caspasa-3 y Bax, que la frecuencia de fenómenos apoptóticos en el LPO es baja, y que no existen diferencia cuantitativas entre las diferentes formas clínicas de la enfermedad (2, 35-37, 40).

Este comportamiento celular, en el que las células desarrollan mecanismos para evadir la apoptosis y, por consiguiente, evitar la regulación de su ciclo biológico, crea un sustrato en el epitelio sobre el que es más sencillo que se desarrolle el cáncer $(35,36)$.

\section{Respuesta proliferativa en el LPO}

La mayoría de los estudios hablan de un aumento en la tasa de proliferación de las células epiteliales en el
LPO, y esto también podría ser un argumento de peso en la justificación del potencial de cancerización de esta enfermedad (36).

\section{Papel de la proteína p53}

Se ha constatado una sobreexpresión de esta proteína en las lesiones de LPO, aunque se desconoce el mecanismo que lo desencadena. Sin embargo, se ha visto que la tasa de malignización del LPO no es consistente con la elevada expresión de la proteína en estos pacientes, lo cual podría cobrar sentido en caso de existir formas mutadas de esta proteína (36).

En cualquiera de los casos, existen distintas vías que justifiquen la plausibilidad biológica de la relación, por eso, a pesar de la falta de consenso a la hora de dar datos certeros que corroboren la transformación maligna del LPO, conviene manejar esta enfermedad como potencialmente cancerizable.

\section{TRATAMIENTO}

El liquen plano es una enfermedad de carácter crónico, por lo que los posibles tratamientos no se aplican con la idea de "curar", sino más bien se pretende controlar con ellos las fases agudas o los picos de exacerbación (23).

Una higiene oral excelente se cree que puede ayudar a reducir la severidad de los síntomas, pero, dado el grado de disconfort y dolor que provocan ciertas formas clínicas de LPO, puede que resulte difícil para los pacientes mantener una correcta higiene.

El tratamiento en principio sólo está encaminado a reducir la duración y la severidad de los síntomas, por lo que en principio las formas las formas blancas o asintomáticas sólo requieren observación y vigilancia. Ahora bien, antes de comenzar con cualquiera de las opciones de tratamiento es necesario confirmar el diagnóstico histológicamente, y descartar otras patologías de presentación clínica similar al LPO, pero que podrían complicarse con el tratamiento de éste (23).

Las posibilidades que existen para el tratamiento del liquen son: 


\section{Corticoesteroides}

\section{Tópicos}

Suelen emplearse en orabases durante periodos de tiempo prolongados, pero a día de hoy están considerados como el tratamiento de elección (1). La literatura recoge que son seguros si se emplean de forma tópica durante un máximo de 6 meses, pero el peligro de supresión adrenal hace necesaria la supervisión del tratamiento (43). El efecto secundario más comúnmente observado es la aparición de candidiasis secundarias, para las cuales suele ser necesario el tratamiento con antifúngi$\cos (1)$.

En general, el tratamiento debería empezar con un agente tópico de elevada potencia, para poder obtener una respuesta rápida. Los pacientes deben aplicarse el preparado varias veces al día e intentar mantenerlos en contacto con la lesión durante un periodo prolongado de tiempo. A medida que las lesiones evolucionan favorablemente y van remitiendo los síntomas, debe irse reduciendo la potencia del corticosteroide empleado. En el momento en que los síntomas estén perfectamente controlados debe interrumpirse el tratamiento (22).

\section{Sistémicos}

Existen otros autores que sugieren que la terapia con corticoides sistémicos pueda resultar en un mejor control de las formas agudas de presentación (44). Por ello, se ha comparado el tratamiento con corticoides tópicos y sistémicos con la terapia a base de corticoides tópicos exclusivamente. Sin embargo, no se han encontrado diferencias estadísticamente significativas, no debiéndose olvidar además los efectos secundarios que el tratamiento sistémico es capaz de provocar. El tratamiento sistémico debería reservarse para aquellos pacientes con un LPO recalcitrante, en forma erosiva o atrófica, en el que el tratamiento tópico no está haciendo efecto, o en el que la extensión sistémica del LPO complica su manejo tópico (45).

En principio los corticoides tópicos deben prescribirse por durante dos semanas, periodo en el que debería observarse cierta respuesta. De no ser así y tenerse que optar por un tratamiento prolongado, la dosis de corticoesteroides debe ir reduciéndose paulatinamente, para evitar el desencadenamiento de una crisis supradrenal (23).

\section{Inyecciones intralesionales}

En casos de lesiones con baja respuesta también pueden inyectarse subcutáneamente los corticoides (23). El problema fundamental de esta terapia recae en su efectividad limitada y en lo dolorosas que resultan estas inyecciones para el paciente.

\section{Otros agentes tópicos}

\section{Tacrólimus}

Se trata de un inmunosupresor no esteroideo, de potencia hasta 100 veces superior a la de la ciclosporina (1). Se ha comparado su eficacia frente a la de los retinoides tópicos, y se ha visto que los preparados de Tacrólimus al $0,1 \%$ resultan más efectivos que el propionato de clobetasol al $0,05 \%$ en el tratamiento del LPO (46). Sin embargo, los autores reconocen en este estudio que para poder defender el uso de inmunosupresores para el tratamiento de esta enfermedad hacen falta todavía estudios llevados a cabo en poblaciones más grandes.

Aunque en principio podría parecer que este fármaco tiene menor número que efectos secundarios que los corticoides, el tacrólimus no está exento de complicaciones. De hecho, se ha visto que acelera la carcinogénesis en ratones, por lo que su uso está restringido (47).

Aunque en la mayoría de casos los inmunosupresores se han indicado como tratamiento tópico, también se han empleado sistémicamente. Sin embargo existe poca literatura que evalúe su eficacia (1).

\section{Tratamiento quirúrgico}

Se ha recomendado el tratamiento mediante exéresis quirúrgica en casos de placas aisladas, o erosiones que no curan con el tratamiento farmacológico. 


\begin{tabular}{|l|c|c|}
\hline \multicolumn{2}{|c|}{ TABLA 7 } \\
\hline Tratamiento & Mec. de acción & Inconvenientes \\
\hline Retinoides & $\begin{array}{c}\text { Inhibición de citoquinas } \\
\text { inductoras de células T }\end{array}$ & $\begin{array}{c}\text { Mal sabor, sensación de quemazón, } \\
\text { alto coste }\end{array}$ \\
\hline Ciclosporina & Disminuye la queratinización & $\begin{array}{c}\text { Queilitis, prurito, sequedad mucosas, } \\
\text { elevación de transaminasas }\end{array}$ \\
\hline Tacrólimus & Inmunosupresor & Irritación local \\
\hline Radiación U.V. & & $\begin{array}{c}\text { Náuseas, mareos, parestesia. } \\
\text { Posible papel oncogénico }\end{array}$ \\
\hline
\end{tabular}

Se dice que el manejo quirúrgico de las lesiones, además de proporcionar un excelente tejido para el análisis histopatológico, es capaz de curar lesiones localizadas. Sin embargo, no existe suficiente evidencia que lo corrobore (1).

Para el manejo quirúrgico se han propuesto tanto los láseres como el de dióxido de carbono, la terapia fotodinámica, y la criocirugía (3).

Tras la revisión de este apartado, parece que los corticoesteroides tópicos podrían considerarse el tratamiento de elección, sin embargo la evidencia que lo avala tampoco es sólida (23).

\section{Tratamiento de la gingivitis descamativa}

Dada la sintomatología asociada a la gingivitis descamativa, se han evaluado tratamientos específicos para su remisión. La terapia que ha probado ser eficaz y segura consiste en la aplicación de una orabase de propionato de clobetasol $0,05 \%$, asociado a $100.000 \mathrm{UI} / \mathrm{cc}$ de nistatina en férulas, durante $5 \mathrm{mi}$ nutos, tres veces al día (48). Por medio de este tratamiento se ha corroborado la desaparición del cuadro en un $100 \%$ de los casos.

Sea cual sea la elección de tratamiento siempre es conveniente llevar a cabo una estrategia de revisiones, para garantizar el temprano diagnóstico de cualquier cambio o transformación maligna de las lesiones. En el gráfico 2 se muestra un protocolo de cómo puede estructurarse esta estrategia de revisiones periódicas.

\section{CONCLUSIÓN}

El LPO es una enfermedad mucocutánea de cierta relevancia en nuestros días, en parte por la frecuencia con ésta aparece, pero también por su potencial de malignización (49). Este hecho, aunque aún se encuentra en revisión, merece una atención especial, dada la sencillez de su identificación para el odontólogo, y la rentabilidad desde todos los puntos de vista del diagnóstico certero.

El armamento terapéutico del que disponemos es amplio pero no está específicamente dirigido, por lo cual, además de por el hecho de tratarse de una enfermedad de carácter crónico, no está indicado su empleo más que en aquellos casos con sintomatología aguda. En el resto de casos, lo que sí está indicado es el seguimiento protocolizado de las lesiones, y el control minucioso.

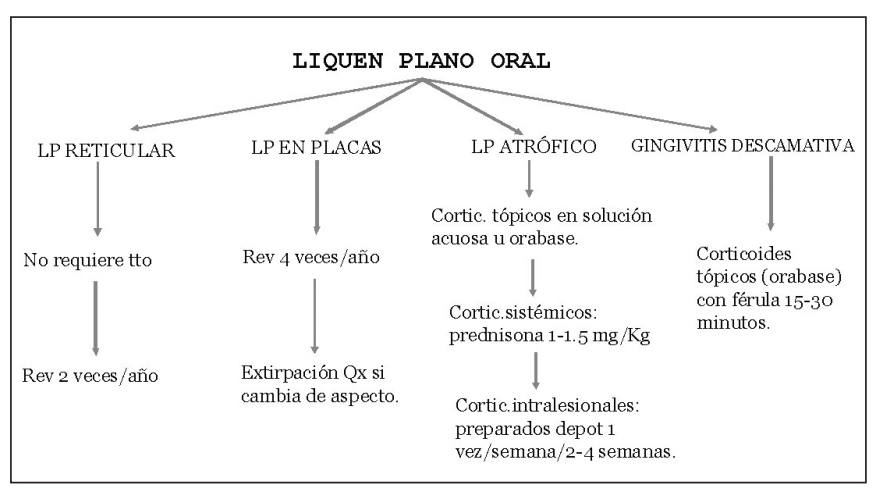

Gráfico 2. 


\section{BIBLIOGRAFÍA}

1. Scully C, Carrozzo M. Oral mucosal disease: Lichen planus. The British journal of oral $\mathcal{E}$ maxillofacial surgery. 2008 Jan;46(1):15-21.

2. Gonzalez-Moles MA, Scully C, Gil-Montoya JA. Oral lichen planus: controversies surrounding malignant transformation. Oral diseases. 2008 Apr;14(3):229-43.

3. Al-Hashimi I, Schifter M, Lockhart PB, Wray D, Brennan M, Migliorati CA, et al. Oral lichen planus and oral lichenoid lesions: diagnostic and therapeutic considerations. Oral surgery, oral medicine, oral pathology, oral radiology, and endodontics. 2007 Mar;103 Suppl:S25 e1-12.

4. Pentenero M, Broccoletti R, Carbone M, Conrotto $\mathrm{D}$, Gandolfo $\mathrm{S}$. The prevalence of oral mucosal lesions in adults from the Turin area. Oral diseases. 2008 May;14(4):356-66.

5. Myers SL, Rhodus NL, Parsons HM, Hodges JS, Kaimal S. A retrospective survey of oral lichenoid lesions: revisiting the diagnostic process for oral lichen planus. Oral surgery, oral medicine, oral pathology, oral radiology, and endodontics. 2002 Jun;93(6):676-81.

6. Jainkittivong A, Aneksuk V, Langlais RP. Oral mucosal conditions in elderly dental patients. Oral diseases. 2002 Jul;8(4):218-23.

7. Ingafou M, Leao JC, Porter SR, Scully C. Oral lichen planus: a retrospective study of 690 British patients. Oral diseases. 2006 Sep;12(5): 463-8.

8. Cerero R, Garcia-Pola MJ. (Management of oral lichen planus). Med Oral. 2004 Mar-Apr;9(2): 124.

9. Garcia-Pola MJ, Huerta G. Ansiety as an etiologic factor in oral lichen planus. Med Oral. 2000 Jan; 5(1):7-13.

10. Katta R. Lichen planus. American family physician. 2000 Jun 1;61(11):3319-24, 27-8.

11. Laeijendecker R, Dekker SK, Burger PM, Mulder PG, Van Joost T, Neumann MH. Oral lichen planus and allergy to dental amalgam restorations. Archives of dermatology. 2004 Dec; 140(12):1434-8.

12. Pezelj-Ribaric S, Prpic J, Miletic I, Brumini G, Simunovic Soskic M, Anic I. Association between oral lichenoid reactions and amalgam restorations. J Eur Acad Dermatol Venereol. 2008 Apr 2.

13. Dunsche A, Kastel I, Terheyden H, Springer IN, Christophers E, Brasch J. Oral lichenoid reactions associated with amalgam: improvement after amalgam removal. The British journal of dermatology. 2003 Jan;148(1):70-6.

14. Issa Y, Duxbury AJ, Macfarlane TV, Brunton PA. Oral lichenoid lesions related to dental restorative materials. British dental journal. 2005 Mar 26;198(6):361-6; disussion 549; quiz 372.

15. de Mattos Camargo Grossmann S, de Aguiar MC, Teixeira R, do Carmo MA. Oral lichen planus and chronic hepatitis C: a controversial association. American journal of clinical pathology. 2007 May; 127(5):800-4.

16. Lodi G, Scully C, Carrozzo M, Griffiths M, Sugerman PB, Thongprasom K. Current controversies in oral lichen planus: report of an international consensus meeting. Part 1 . Viral infections and etiopathogenesis. Oral surgery, oral medicine, oral pathology, oral radiology, and endodontics. 2005 Jul;100(1):40-51.

17. Carrozzo M. Oral diseases associated with hepatitis $C$ virus infection. Part 2: lichen planus and other diseases. Oral diseases. 2008 Apr;14(3): 217-28.

18. Khan A, Farah CS, Savage NW, Walsh LJ, Harbrow DJ, Sugerman PB. Th1 cytokines in oral lichen planus. J Oral Pathol Med. 2003 Feb;32(2):77-83.

19. Rhodus NL, Cheng B, Ondrey F. Th1/Th2 cytokine ratio in tissue transudates from patients with oral lichen planus. Mediators of inflammation. 2007;2007:19854.

20. Rhodus NL, Cheng B, Bowles W, Myers S, Miller L, Ondrey F. Proinflammatory cytokine levels in 
saliva before and after treatment of (erosive) oral lichen planus with dexamethasone. Oral diseases. 2006 Mar; 12(2):112-6.

21. Romero MA, Seoane J, Varela-Centelles P, DizDios P, Garcia-Pola MJ. Prevalence of diabetes mellitus amongst oral lichen planus patients. Clinical and pathological characteristics. Med Oral. 2002 Mar-Apr;7(2):121-9.

22. Eisen D, Carrozzo M, Bagan Sebastian JV, Thongprasom K. Number V Oral lichen planus: clinical features and management. Oral diseases. 2005 Nov; 11(6):338-49.

23. Edwards PC, Kelsch R. Oral lichen planus: clinical presentation and management. Journal (Canadian Dental Association). 2002 Sep;68 (8):494-9.

24. Camacho-Alonso F, Lopez-Jornet P, BermejoFenoll A. Gingival involvement of oral lichen planus. Journal of periodontology. 2007 Apr;78(4):640-4.

25. Petruzzi M, De Benedittis M, Pastore L, Grassi FR, Serpico R. Peno-gingival lichen planus. Journal of periodontology. 2005 Dec;76(12): 2293-8.

26. Mobio S, Badran Z, Tessier MH, Giumelli B, Soueidan A. (Desquamative gingivitis). Revue belge de medecine dentaire. 2007;62(3):130-40.

27. Markopoulos AK, Antoniades D, Papanayotou P, Trigonidis G. Desquamative gingivitis: a clinical, histopathologic, and immunologic study. Quintessence Int. 1996 Nov;27(11):763-7.

28. Yih WY, Maier T, Kratochvil FJ, Zieper MB. Analysis of desquamative gingivitis using direct immunofluorescence in conjunction with histology. Journal of periodontology. 1998 Jun;69(6):678-85.

29. van der Meij EH, van der Waal I. Lack of clinicopathologic correlation in the diagnosis of oral lichen planus based on the presently available diagnostic criteria and suggestions for modifications. J Oral Pathol Med. 2003 Oct;32 (9):507-12.
30. Onofre MA, Sposto MR, Navarro CM, Motta ME, Turatti E, Almeida RT. Potentially malignant epithelial oral lesions: discrepancies between clinical and histological diagnosis. Oral diseases. 1997 Sep;3(3):148-52.

31. Kramer IR, Lucas RB, Pindborg JJ, Sobin LH. Definition of leukoplakia and related lesions: an aid to studies on oral precancer. Oral surgery, oral medicine, and oral pathology. 1978 Oct;46 (4):518-39.

32. van der Meij EH, Reibel J, Slootweg PJ, van der Wal JE, de Jong WF, van der Waal I. Interobserver and intraobserver variability in the histologic assessment of oral lichen planus. J Oral Pathol Med. 1999 Jul;28(6):274-7.

33. van der Meij EH, Schepman KP, Plonait DR, Axell $\mathrm{T}$, van der Waal I. Interobserver and intraobserver variability in the clinical assessment of oral lichen planus. J Oral Pathol Med. 2002 Feb;31(2): 95-8.

34. Bloor BK, Malik FK, Odell EW, Morgan PR. Quantitative assessment of apoptosis in oral lichen planus. Oral surgery, oral medicine, oral pathology, oral radiology, and endodontics. 1999 Aug;88(2):187-95.

35. Bascones-Ilundain C, Gonzalez-Moles MA, Esparza-Gomez G, Gil-Montoya JA, Bascones-Martinez A. Importance of apoptotic mechanisms in inflammatory infiltrate of oral lichen planus lesions. Anticancer research. 2006 Jan-Feb;26 (1A):357-62.

36. Gonzalez-Moles MA, Bascones-Ilundain C, Gil Montoya JA, Ruiz-Avila I, Delgado-Rodriguez M, Bascones-Martinez A. Cell cycle regulating mechanisms in oral lichen planus: molecular bases in epithelium predisposed to malignant transformation. Archives of oral biology. 2006 Dec;51(12):1093-103.

37. Bascones C, Gonzalez-Moles MA, Esparza G, Bravo M, Acevedo A, Gil-Montoya JA, et al. Apoptosis and cell cycle arrest in oral lichen planus Hypothesis on their possible influence on its 
malignant transformation. Archives of oral biology. 2005 Oct;50(10):873-81.

38. Gonzalez-Moles MA, Gil-Montoya JA, Ruiz-Avila I, Esteban F, Bascones-Martinez A. Differences in the expression of p53 protein in oral lichen planus based on the use of monoclonal antibodies DO7 and pAb 240. Oral oncology. 2008 May;44(5): 496-503.

39. van der Meij EH, Schepman KP, Smeele LE, van der Wal JE, Bezemer PD, van der Waal I. A review of the recent literature regarding malignant transformation of oral lichen planus. Oral surgery, oral medicine, oral pathology, oral radiology, and endodontics. 1999 Sep;88(3):307-10.

40. Bascones-Ilundain C, Gonzalez-Moles MA, Campo-Trapero J, Gil-Montoya JA, Esparza-Gomez GC, Cano-Sanchez J, et al. No differences in caspase-3 and Bax expression in atrophic-erosive vs. reticular oral lichen planus. J Eur Acad Dermatol Venereol. 2008 Feb;22(2):204-12.

41. Mignogna MD, Lo Russo L, Fedele S. Gingival involvement of oral lichen planus in a series of 700 patients. Journal of clinical periodontology. 2005 Oct;32(10):1029-33.

42. Tanda N, Mori S, Saito K, Ikawa K, Sakamoto S. Expression of apoptotic signaling proteins in leukoplakia and oral lichen planus: quantitative and topographical studies. J Oral Pathol Med. 2000 Sep;29(8):385-93.

43. Carbone M, Conrotto D, Carrozzo M, Broccoletti R, Gandolfo S, Scully C. Topical corticosteroids in association with miconazole and chlorhexidine in the long-term management of atrophicerosive oral lichen planus: a placebo-controlled and comparative study between clobetasol and fluocinonide. Oral diseases. 1999 Jan;5 (1):44-9.

44. Lozada-Nur F, Miranda C. Oral lichen planus: topical and systemic therapy. Seminars in cutaneous medicine and surgery. 1997 Dec;16(4):295-300.
45. Carbone M, Goss E, Carrozzo M, Castellano S, Conrotto D, Broccoletti R, et al. Systemic and topical corticosteroid treatment of oral lichen planus: a comparative study with long-term follow-up. J Oral Pathol Med. 2003 Jul;32(6): 323-9.

46. Corrocher G, Di Lorenzo G, Martinelli N, Mansueto P, Biasi D, Nocini PF, et al. Comparative effect of tacrolimus $0.1 \%$ ointment and clobetasol $0.05 \%$ ointment in patients with oral lichen planus. Journal of clinical periodontology. 2008 Mar;35(3):244-9.

47. Niwa Y, Terashima T, Sumi H. Topical application of the immunosuppressant tacrolimus accelerates carcinogenesis in mouse skin. The British journal of dermatology. 2003 Nov; 149 (5):960-7.

48. Gonzalez-Moles MA, Ruiz-Avila I, RodriguezArchilla A, Morales-Garcia P, Mesa-Aguado F, Bascones-Martinez A, et al. Treatment of severe erosive gingival lesions by topical application of clobetasol propionate in custom trays. Oral surgery, oral medicine, oral pathology, oral radiology, and endodontics. 2003 Jun;95(6):688-92.

49. Bascones-Ilundain C, Gonzalez-Moles MA, Carrillo de Aobornoz A, Bascones-Martínez A. Liquen plano oral (I). Aspectos clínicos, etiopatogénicos y epidemiológicos. Av. Odontoestomatol 2006; 22(1):11-19.

50. Bascones-llundain C, Gonzalez-Moles MA, Carrillo de Aobornoz A, Bascones-Martínez A. Liquen plano oral (II). Aspectos clínicos, etiopatogénicos y epidemiológicos. Av. Odontoestomatol 2006;22(1):21-31.

\section{CORRESPONDENCIA}

Paula Matesanz Pérez

C/Castilla La Mancha, $11^{\mathrm{a} A}$

28750 Madrid

paula_matesanz@yahoo.es 